\section{Pathways in opposition}

Microbes are recognized via the activation of multiple receptors and pathways with potentially overlapping, synergistic or oppositional effects. In PLoS Pathogens, Smale and colleagues assess the early transcriptional response of macrophages to Staphylococcus aureus to understand the contribution of and possible interactions between the TLR and STING pathways. Almost the entire early transcriptional response $(<4 \mathrm{~h})$ to live $S$. aureus is accounted for by these two pathways, with the remainder being driven by hypoxia-pathway signaling. However, TLR signaling is responsible for essentially the entire early transcriptional response to heat-killed bacteria. When assessed in isolation through the use of knockouts, the STING and TLR pathways trigger substantially overlapping transcriptional patterns, albeit with some differences in magnitude and kinetics. However, in an S. aureus skin-infection model, the STING and TLR pathways have opposing roles, with the former diminishing protection dependent on the cyokine IL-1.

PLoS Pathog. (13 Jul 2017) doi:10.1371/journal.ppat. 1006496

\section{The eyes have it}

Commensal microbes colonize mucosal surfaces throughout the body, but whether there is a resident population of microbes present on the ocular surface has been controversial. In Immunity, Caspi and colleagues establish that Cornyebacterium mastitidis is a de facto resident microbe present on mouse conjunctiva that 'tunes' the local immune response. Several other bacteria tested, including other Cornyebacterium species and Staphylococcus epidermidis, fail to colonize the conjunctiva after inoculation. The presence of $C$. mastitidis results mainly in the activation and population expansion of ocular IL-17A $\mathrm{A}^{+} \gamma \delta$ T cells, which in turn supports the induction of anti-microbial peptides and the recruitment of neutrophils to the conjunctiva. Mice lacking C. mastitidis have fewer conjunctival IL-17A $\mathrm{A}^{+} \gamma \delta \mathrm{T}$ cells and greater vulnerability to infection of the eye with Candida albicans or Pseudomonas aeruginosa. C. mastitidis is therefore a specialized commensal resident in the conjunctiva that can mediate localized protection through the induction of IL-17A production. $\quad Z F$ Immunity 47, 148-159 (2017)

\section{Rodent hepatitis $\mathrm{C}$ model}

In vivo studies of immune responses to infection with hepatitis $\mathrm{C}$ virus (HCV) have been hampered by the lack of suitable small animal models. In Science, Billerbeck et al. characterize immune responses in mice to an HCV-related hepacivirus originally found in street rats. Wild-type mice develop acute liver infection that can be cleared within several weeks, whereas immunocompromised mice establish chronic viral infection. Interferon and CD4+ $\mathrm{T}$ cell help are necessary for viral clearance, as mice transiently depleted of $\mathrm{CD}^{+}$cells develop chronic infection despite the generation of effector $\mathrm{CD}^{+} \mathrm{T}$ cells. Whereas clearance of acute infection is accompanied by substantial liver damage, chronic infection elicits mild liver inflammation and more intrahepatic regulatory T cells and exhausted CD8+ T cells. Use of this mouse model should enhance efforts to develop effective therapies and vaccines directed against HCV.

Science 357, 204-208 (2017)

\section{Dopamine in germinal centers}

Follicular helper $\mathrm{T}$ cell $\left(\mathrm{T}_{\mathrm{FH}}\right.$ cell)-B cell interactions in germinal centers lead to the generation of long-lived plasma cells that produce high-affinity antibodies. In Nature, Papa et al. show that human $\mathrm{T}_{\mathrm{FH}}$ cells express enzymes necessary for production of the neurotransmitter dopamine, which is released after cognate interactions with follicular B cells that express the dopamine receptor DRD1. This bidirectional interaction leads to upregulation of surface expression of the ligands ICOS-L on B cells and CD40L on $\mathrm{T}_{\mathrm{FH}}$ cells, which enhances the formation of $\mathrm{T}$ cell-B cell synapses. Curiously, mouse follicular lymphocytes do not display similar dopamine-dependent signaling interactions. Simulation experiments suggest that dopamine signaling might speed germinal-center interactions and enhance plasma-cell generation. Testing of this hypothesis, however, awaits the development of in vivo models.

Nature 547, 318-323 (2017)

$L A D$

\section{Losing IFN}

Chronic oxidative injury triggers epigenetic reprogramming of the lung epithelial barrier known as 'epithelial-mesenchymal transition' (EMT), as part of the mucosal repair response mediated by the cytokine TGF- $\beta$. In Nature Microbiology, Brasier and colleagues show that EMT silences the production of protective type I and type III interferons and promotes enhanced viral replication in epithelial cells during infection with rhinovirus or respiratory syncytial virus. In human small-airway epithelial cells, EMT is associated with diminished expression of the transcription factor IRF1 and less binding of IRF1 and IRF7 to the promoters of IFNB1 and IFNL1 (which encode interferons). TGF- $\beta$-driven EMT induces upregulation of the transcription factor ZEB1, which represses IRF1 by inducing the accumulation of repressive epigenetic marks and decreases the expression of IFNB1 and IFNL1. These observations provide a mechanistic basis for the observation that asthma is associated with enhanced susceptibility to pulmonary infection.

Nat. Microbiol. (5 June 2017) doi:10.1038/nmicrobiol.2017.86

\section{Amplifying senescence}

Senescent cells undergo permanent cell-cycle arrest and secrete inflammatory factors, a phenotype referred to as the 'senescence-associated secretory phenotype' (SASP). In Nature Cell Biology, Ablasser and colleagues show that the DNA-sensing pathway cGAS-STING regulates the production of SASP factors and promotes senescence in a paracrine way. Mouse embryonic fibroblasts deficient in cGAS or STING do not undergo proliferative arrest and produce less of the cytokines IL- 6 and TNF and chemokine CXCL10 in culture, a phenotype 'rescued' by conditioned medium from wild-type senescent cells or by recombinant interferon- $\beta$. cGAS localizes together with the chromatin fragments that appear at the nuclear-cytoplasmic interface of senescent nuclei. In vivo, cGAS- and STING-deficient mice show less upregulation of SASP factors such as IL-6, CXCL10, Cdkn2a and p21 after irradiation or stimulation with oncogenic transposable elements. Because SASP is a late event in the development of senescence, cGAS-STING might amplify inflammation in cells already on the senescence track. IV Nat. Cell Biol. (31 July 2017) doi:10.1038/ncb3586 